\title{
Prediction of clinical outcome in subacute subarachnoid hemorrhage using diffusion tensor imaging
}

\author{
Isabel Fragata, MD, MSc, ${ }^{1,2}$ Marta Alves, MSc, ${ }^{3}$ Ana Luísa Papoila, PhD, ${ }^{3,4}$ Patrícia Ferreira, MD, ${ }^{5}$ \\ Ana Paiva Nunes, MD, ${ }^{5}$ Nuno Canto Moreira, MD, PhD, ${ }^{6}$ and Patrícia Canhão, MD, $\mathrm{PhD} 7,8$

\begin{abstract}
${ }^{1}$ Neuroradiology Department; ${ }^{3}$ Centro de Investigação; ${ }^{5}$ Cerebrovascular Unit, Centro Hospitalar Lisboa Central; " ${ }^{\text {Biostatistics }}$ Department, ${ }^{2}$ Nova Medical School/Faculdade de Ciências Médicas, Universidade Nova de Lisboa; ${ }^{7}$ Neurology Department, Centro Hospitalar Lisboa Norte; ${ }^{8}$ Faculdade de Medicina, University of Lisbon, Portugal; and ${ }^{6}$ Department of Neuroradiology, Karolinska University Hospital, Stockholm, Sweden
\end{abstract}

\begin{abstract}
OBJECTIVE Clinical outcome in nontraumatic subarachnoid hemorrhage (SAH) is multifactorial and difficult to predict. Diffusion tensor imaging (DTI) findings are a prognostic marker in some diseases such as traumatic brain injury. The authors hypothesized that DTI parameters measured in the subacute phase of SAH can be associated with a poor clinical outcome.
\end{abstract}

METHODS Diffusion tensor imaging was prospectively performed in 54 patients at 8-10 days after nontraumatic SAH. Logistic regression analysis was performed to evaluate the association of fractional anisotropy (FA) and apparent diffusion coefficient (ADC) values with a poor clinical outcome (modified Rankin Scale score $\geq 3$ ) at 3 months.

RESULTS At 8-10 days post-SAH, after adjusting for other variables associated with a poor outcome, an increased $\mathrm{ADC}$ at the frontal centrum semiovale was associated with a poor prognosis (OR estimate 1.29, 95\% $\mathrm{Cl} 1.04-1.60, \mathrm{p}$ $=0.020$ ). Moreover, an increase of 0.1 in the FA value at the corpus callosum at $8-10$ days after SAH corresponded to $66 \%$ lower odds of having a poor outcome $(p=0.002)$.

CONCLUSIONS Decreased FA and increased ADC values in specific brain regions were independently associated with a poor clinical outcome after SAH. This preliminary exploratory study supports a potential role for DTI in predicting the outcome of SAH.

https://thejns.org/doi/abs/10.3171/2017.10.JNS171793

KEYWORDS subarachnoid hemorrhage; diffusion tensor imaging; clinical outcome; vascular disorders

$\mathrm{S}$ PONTANEOUS subarachnoid hemorrhage (SAH) has high mortality and morbidity. ${ }^{19}$ Most prognostic factors are present on admission, and others develop a few days later, for example, hydrocephalus or delayed cerebral ischemia (DCI)..$^{15,17,29-31}$

Magnetic resonance imaging (MRI), in particular diffusion tensor imaging (DTI), is a sensitive noninvasive tool for detecting neuronal injury. The measurement of parameters such as fractional anisotropy (FA) and apparent diffusion coefficient (ADC) allows for indirect evaluation of white matter integrity and the presence of cytotoxic edema, respectively. ${ }^{26}$ Diffusion tensor imaging evaluation has proved to be a valuable tool for predicting prognosis in traumatic brain injury, in which reductions in FA have been associated with an unfavorable long-term outcome, ${ }^{40}$ as well as in small vessel disease. ${ }^{27}$

Changes in FA values over time have been reported in patients with SAH, especially in those who develop DCI. ${ }^{10}$ Although a reduction in ADC values in the cerebral white matter has been described in the vasospasm period, ${ }^{4}$ other studies have found an increase in ADC values in normalappearing white matter in both the acute ${ }^{38}$ and the sub-

ABBREVIATIONS ADC = apparent diffusion coefficient; $A U C=$ area under the ROC curve; $D C l=$ delayed cerebral ischemia; $D T I=$ diffusion tensor imaging; $D W I=$ diffusion-weighted imaging; FA = fractional anisotropy; GCS = Glasgow Coma Scale; HH = Hunt and Hess; MRI = magnetic resonance imaging; $m R S=$ modified Rankin Scale; PLIC = posterior limb of internal capsule; ROC = receiver operating characteristic; ROI = region of interest; $S A H$ = subarachnoid hemorrhage; WFNS = World Federation of Neurosurgical Societies.

SUBMITTED July 22, 2017. ACCEPTED October 16, 2017.

INCLUDE WHEN CITING Published online April 13, 2018; DOI: 10.3171/2017.10.JNS171793. 
acute $^{25}$ phase, suggesting the presence of vasogenic edema. However, the relation of these changes to prognosis has been less studied: one analysis has suggested that very early changes in DTI parameters are independent predictors of outcome, ${ }^{9}$ and another study has supported a potential role for DTI as a predictor of late mortality in patients with severe $\mathrm{SAH} .^{33}$

In this study, we hypothesized that DTI parameters measured in the subacute phase of SAH may add a prognostic indication for clinical outcome in SAH patients.

\section{Methods \\ Sample}

Patients with acute spontaneous SAH admitted to our institution between May 2013 and November 2014 were consecutively enrolled in a prospective cohort study. Institutional review board approval was obtained. Patients were included in the study if they 1) had an age $>18$ years, 2) had acute nontraumatic SAH diagnosed by CT or lumbar puncture, 3) had imaging studies (CT or MRI) performed within the first 72 hours of SAH onset, and 4) provided informed consent either from themselves or a legal representative. Exclusion criteria were as follows: poor clinical condition (Glasgow Coma Scale [GCS] score 3), pregnancy, renal insufficiency, any contraindication to performing MRI, or time for onset of SAH unknown. For the current analysis, we included patients who had undergone MRI at 8-10 days after SAH.

\section{Clinical and Imaging Data}

Demographic and clinical presentation data were collected from the patient medical records, such as hydrocephalus, vasospasm on transcranial Doppler ultrasonography, DCI, aneurysm, and surgical and/or endovascular treatment. The GCS, ${ }^{34}$ World Federation of Neurosurgical Societies (WFNS) scale, ${ }^{6}$ and Hunt and Hess (HH) scale ${ }^{20}$ were used to assess neurological status at admission. The modified Fisher scale ${ }^{12}$ and the Hijdra scale for cisternal and intraventricular hemorrhage ${ }^{18}$ were used to quantify the amount of blood on admission brain CT. Hydrocephalus was defined as a bicaudate index above the 95th percentile for age, at any time between admission and discharge. ${ }^{35}$ The presence of cerebral vasospasm was defined by a Lindegaard index ${ }^{24}$ above 3 (moderate/severe vasospasm) on transcranial Doppler ultrasonography studies after the 4th day post-SAH. Patients were classified as having DCI if 1) they presented with a new focal neurological deficit and/or a decrease in their level of consciousness not attributable to other causes (for example, hydrocephalus, seizures, metabolic derangement, infection, or sedation), 2) there was a new infarct on follow-up CT or MRI after 4 days after ictus, or 3 ) both 1 and 2.13,36

\section{MRI Protocol}

Magnetic resonance imaging was performed on days 8-10 after SAH. All MRI was performed on the same 1.5-T scanner (Magnetom Avanto, Siemens Medical Systems) with an imaging protocol that included T1, T2, T2*, FLAIR, and DTI sequences. In the DTI sequence, for each of the 20 noncollinear diffusion-sensitizing gradients, we acquired 84 contiguous slices. Imaging parameters were as follows: matrix $128 \times 128$, FOV $230 \mathrm{~mm}$, TE $95 \mathrm{msec}$, TR $2800 \mathrm{msec}$, EPI factor $128, \mathrm{~b}=1000 \mathrm{sec} / \mathrm{mm}^{2}$, NEX 1, and slice thickness $5 \mathrm{~mm}$.

\section{Postprocessing}

Regions of interest (ROIs) of $20 \mathrm{~mm}^{2}$ were selected using OLEA software, at the following locations: frontal centrum semiovale, parietal centrum semiovale, posterior limb of internal capsule (PLIC), lentiform nucleus, thalamus, genu and splenium of corpus callosum, mid pons, and subcortical cerebellar hemispheres, bilaterally and symmetrically (Fig. 1). All measurements were performed by the same observer, who was blinded to the clinical data and had 10 years' experience in neuroradiology. No significant differences were found between the 2 sides, and an average of the left and right ROIs for each anatomical location was analyzed. Mean FA and ADC were measured for each ROI. Whole-brain mean FA and ADC values were calculated from total ROIs.

\section{Outcome}

We aimed to assess the association between DTI measurements and the modified Rankin Scale (mRS) score at 3 months, ${ }^{2}$ as a clinical outcome. The mRS was applied at 3 months via in-person or telephone interview by 2 physicians with experience in stroke (A.P.N., P.F.) and who were blinded to the clinical and MRI data. ${ }^{21}$ Clinical outcome was dichotomized as good (mRS score $<3)$ and poor (mRS score $\geq 3$ ).

\section{Statistical Analysis}

Demographic and clinical characteristics of patients were described as frequencies (percentages), the mean \pm standard deviation, or the median (minimum-maximum range), as appropriate. To compare FA and ADC ROI values between the left and right hemispheres, the Wilcoxon signed-rank test was used. Logistic regression models were used to study the association between DTI parameters and clinical outcome. Discriminative and predictive abilities of the final multivariable models were assessed using the area under the receiver operating characteristic (ROC) curve (AUC) and the Hosmer-Lemeshow test, respectively. The following variables were considered in the univariable analyses: age, sex, GCS score, WFNS grade (I vs II-V), HH grade (I vs II, III, IV-V), modified Fisher grade (2-3 vs 4), cisternal Hijdra grading, ventricular Hijdra grading, SAH location (perimesencephalic vs nonperimesencephalic), vasospasm (yes/no), hydrocephalus (yes/no), DCI (yes/no), treatment (nonaneurysmal SAH, clipped aneurysm, coiled aneurysm), and DTI parameters measured in all ROIs. Variables that attained a p value $\leq$ 0.25 were considered for multivariable analysis.

Generalized additive models were applied to model the association between age and poor clinical outcome. The level of signigicance was considered to be $\alpha=0.05$. Data were analyzed using Stata 13.0 (StataCorp LP) and $\mathrm{R}$ software (R: A language and environment for statistical computing, R Core Team, R Foundation for Statistical Computing, http://www.R-project.org.). 


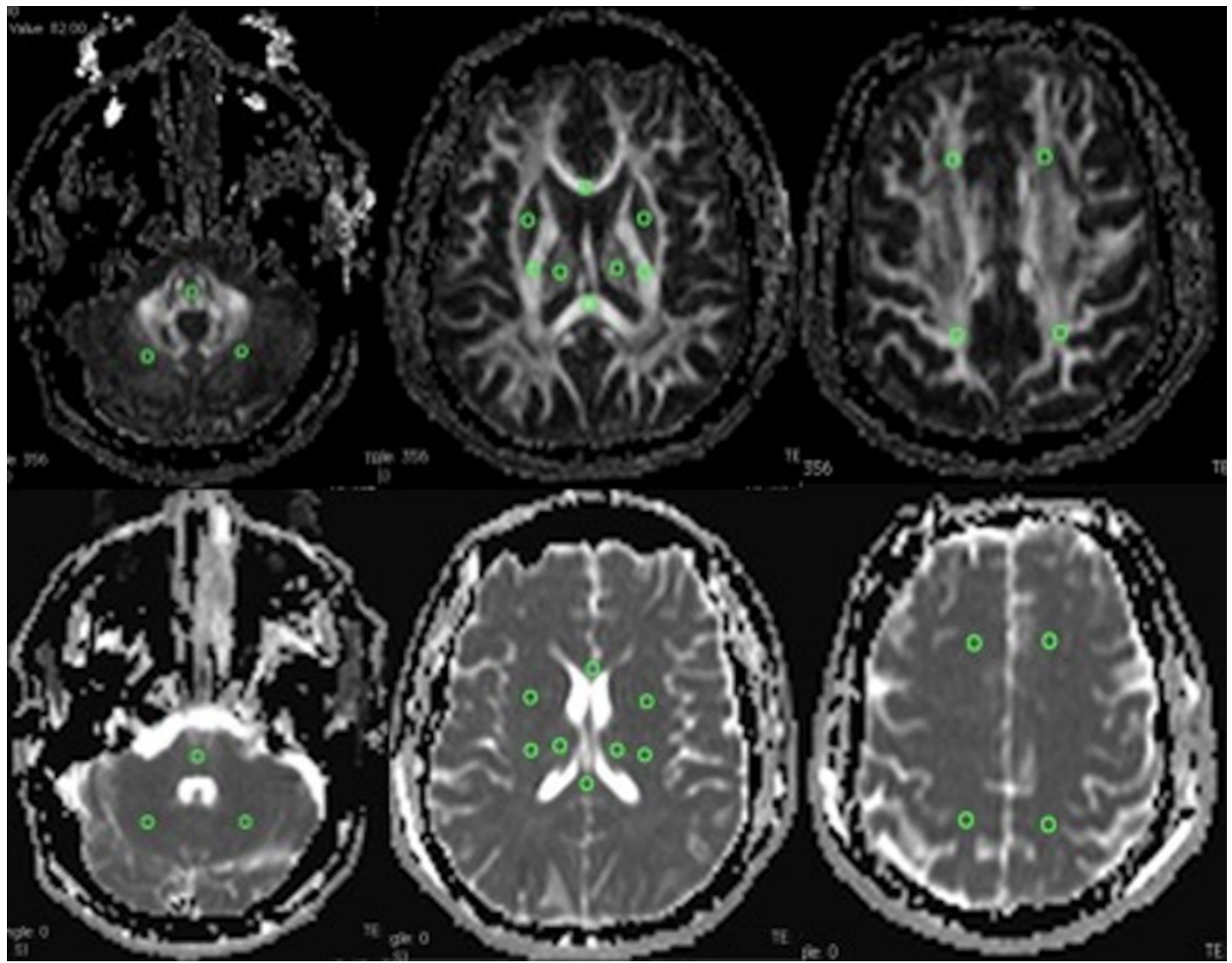

FIG. 1. Transverse FA and ADC maps show ROIs drawn bilaterally in the brain parenchyma. Upper and lower left: Regions of interest in the pons and cerebellar white matter. Upper and lower center: Regions of interest in the lentiform nucleus, thalamus, PLIC, and splenium and genu of corpus callosum. Upper and lower right: Regions of interest in the frontal and parietal centrum semiovale. From Neuroradiology, Evolution of diffusion tensor imaging parameters after acute subarachnoid haemorrhage: a prospective cohort study, 59, 2017, 13-21, Fragata I, Canhão P, Alves M, Papoila AL, Canto-Moreira N. With permission from Springer. Figure is available in color online only.

\section{Results}

\section{Demographic and Clinical Data}

During the inclusion period, 129 patients with nontraumatic SAH were admitted to our institution. Eighty patients fulfilled the inclusion criteria for the prospective cohort. Of these, 10 patients were excluded from the analysis because MRI could not be performed. Another 16 patients were excluded because their images were degraded by artifacts, leaving a total of 54 patients eligible for the current analysis (Fig. 2).

The median patient age was 60 years (range 35-86 years), and $63 \%$ of the patients were female. The median HH grade was II (range I-V), 29 patients had WFNS grade I SAH, and 6 patients had GCS score $<8$ on admission (Table 1). A total of 15 patients $(27.8 \%)$ developed DCI. At 3 months, 15 patients $(28 \%)$ were dependent (mRS score $\geq 3$; (Table 2). The mortality rate at 3 months was $3.7 \%$ (2 patients).

Univariable regression analysis of clinical variables identified age, clinical grade on admission (WFNS grade and GCS score), amount of blood on admission CT (modified Fisher and Hijdra scales), hydrocephalus, aneurysmal
SAH, and SAH location as candidates in the multivariable model (Table 2). A nonlinear association of patient age with poor outcome was identified, as younger and older patients had higher odds of a poor outcome. Accordingly, age was modeled with splines through a generalized additive model (Fig. 3) and was dichotomized into 2 categories: age 42-67 years and ages $<42 />67$ years.

\section{Fractional Anisotropy in Subacute SAH as an Indicator of Poor Clinical Outcome}

Univariable analysis concerning FA (Table 3) yielded the frontal centrum semiovale, parietal centrum semiovale, lentiform nucleus, corpus callosum, subcortical cerebellum, and pons as candidates for the multivariable model (Fig. 4A). An association between whole-brain FA and poor clinical outcome was also found in the univariable analysis (OR $0.41,95 \%$ CI $0.01-0.036, \mathrm{p}=0.004)$ but was not included in the multivariable model to avoid redundancy since whole-brain FA was calculated from the mean of all ROIs.

In the multivariable analysis, only FA at the corpus callosum (OR 0.34, 95\% CI 0.17-0.68, p = 0.002) and age 


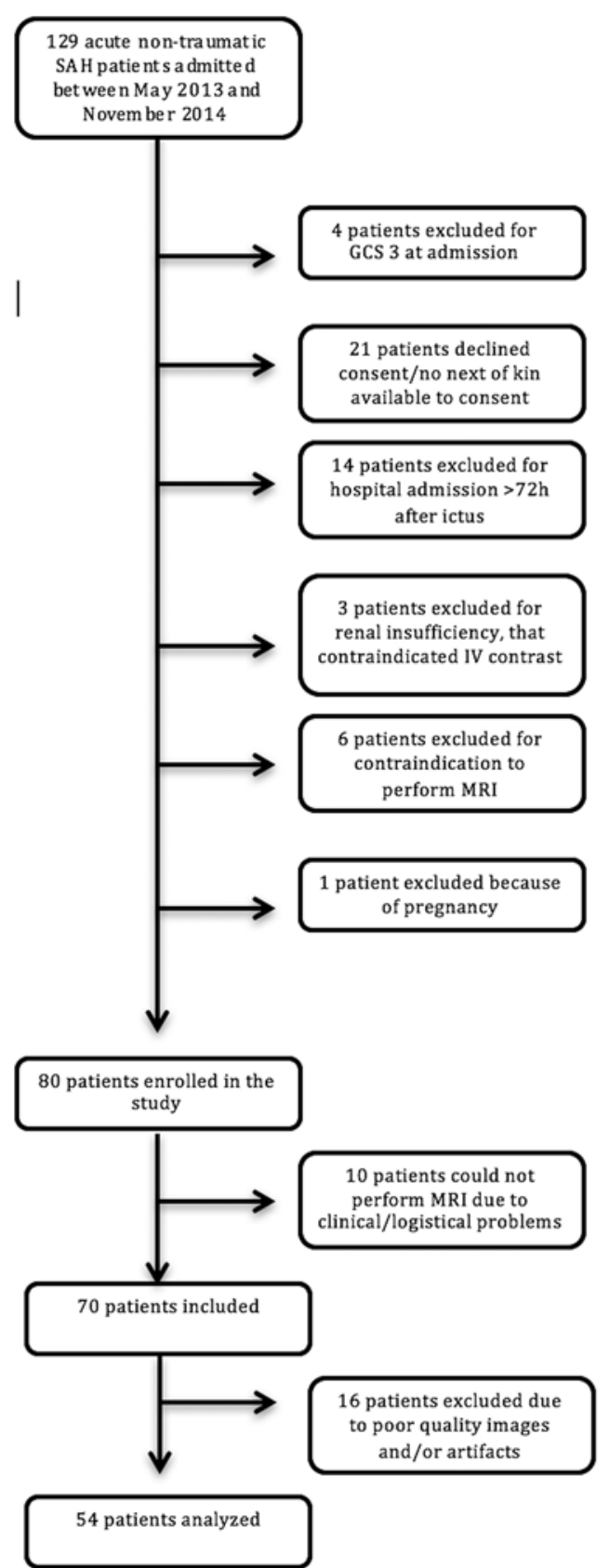

FIG. 2. Flowchart with the number of patients admitted, excluded, and enrolled in the present study. $\mathrm{h}=$ hours; IV = intravenous.

(OR 17.6, 95\% CI 2.31-134.1, $\mathrm{p}=0.006)$ showed an association with poor outcome at 3 months after SAH. For each increase of 0.1 in the FA value measured at the corpus callosum, there was a $66 \%$ reduction in the odds of having a poor clinical outcome. Younger and older patients (age $<42$ and $>67$ years, respectively) had 17-fold increased odds of a poor outcome. The ROC curve had an AUC of 0.88 (95\% CI 0.79-0.98, good discriminative ability), and the Hosmer-Lemeshow goodness-of-fit test showed good calibration $(\mathrm{p}=0.665)$.
TABLE 1. Demographic, clinical, and imaging characteristics of 54 patients with nontraumatic SAH

\begin{tabular}{|c|c|}
\hline Characteristic & Value \\
\hline Median age in yrs (range) & $60(35-86)$ \\
\hline No. of females (\%) & $34(63)$ \\
\hline \multicolumn{2}{|l|}{ No. w/ comorbidities (\%) } \\
\hline Hypertension & $24(44)$ \\
\hline Smoking & $11(20)$ \\
\hline Diabetes & $2(4)$ \\
\hline \multicolumn{2}{|l|}{ Clinical grade at admission } \\
\hline Median GCS score (range) & $15(4-15)$ \\
\hline \multicolumn{2}{|l|}{ WFNS grade (no. [\%]) } \\
\hline 1 & $29(54)$ \\
\hline II & $10(19)$ \\
\hline III & $1(2)$ \\
\hline IV & $9(17)$ \\
\hline V & $5(9)$ \\
\hline \multicolumn{2}{|l|}{ HH grade (no. [\%]) } \\
\hline 1 & $19(35)$ \\
\hline II & $11(20)$ \\
\hline III & $13(24)$ \\
\hline IV & $4(7)$ \\
\hline V & $7(13)$ \\
\hline \multicolumn{2}{|l|}{ Imaging at admission } \\
\hline \multicolumn{2}{|l|}{ Modified Fisher grade (no. [\%]) } \\
\hline 2 & $6(11)$ \\
\hline 3 & $14(26)$ \\
\hline 4 & $34(63)$ \\
\hline \multicolumn{2}{|l|}{ Median Hijdra grade (range) } \\
\hline Cisternal & $14(2-26)$ \\
\hline Ventricular & $2(0-12)$ \\
\hline Aneurysmal SAH (no. [\%]) & $40(74)$ \\
\hline Ant circulation & $33(83)$ \\
\hline Pst circulation & $7(17)$ \\
\hline Nonaneurysmal SAH (no. [\%]) & $14(26)$ \\
\hline Perimesencephalic SAH & $12(86)$ \\
\hline Nonperimesencephalic SAH & $2(14)$ \\
\hline Hydrocephalus (no. [\%]) & $8(15)$ \\
\hline Clipped aneurysm (no. [\%]) & $11(28)$ \\
\hline Coiled aneurysm (no. [\%]) & $30(75)$ \\
\hline
\end{tabular}

Ant $=$ anterior $;$ pst $=$ posterior .

\section{Apparent Diffusion Coefficient in Subacute SAH as an Indicator of Poor Clinical Outcome}

In the univariable analysis for ADC (Table 3), the frontal centrum semiovale, lentiform nucleus, thalamus, PLIC, subcortical cerebellum, and pons were selected for the multivariable model (Fig. 4B). An association between whole-brain ADC and poor clinical outcome was also found in the univariable analysis but again was not included in the multivariable model to avoid redundancy.

Multivariable analysis showed that ADC measured at the frontal centrum semiovale was associated with a poor 
TABLE 2. Univariable regression analysis results for a poor clinical outcome

\begin{tabular}{|c|c|c|c|c|}
\hline Variable & mRS Score $<3$ & mRS Score $\geq 3$ & OR Estimate (95\% Cl) & p Value* \\
\hline No. of patients & 39 & 15 & & \\
\hline Median age in yrs (range) & $53(35-84)$ & $69(35-86)$ & $1.08(1.02,1.13)$ & 0.006 \\
\hline Male sex (no. [\%]) & $15(38.5)$ & $5(33.3)$ & $0.80(0.23,2.80)$ & 0.727 \\
\hline Median GCS score (range) & $15(4-15)$ & $10(4-15)$ & $0.65(0.50,0.83)$ & 0.001 \\
\hline WFNS grades II-V (no. [\%]) & $11(28.2)$ & $14(93.3)$ & $35.64(4.17,304.50)$ & 0.001 \\
\hline \multicolumn{5}{|l|}{$\mathrm{HH}$ grade $($ no. $[\%]) \dagger$} \\
\hline II & $11(28.2)$ & $0(0.0)$ & & \\
\hline III & $8(20.5)$ & $5(33.3)$ & & \\
\hline IV-V & $2(5.1)$ & $9(60.0)$ & & \\
\hline Modified Fisher grade 4 (no. [\%]) & $22(56.4)$ & $13(86.7)$ & $5.02(1.00,25.32)$ & 0.051 \\
\hline \multicolumn{5}{|l|}{ Median Hijdra grade (range) } \\
\hline Cisternal & $12(2-26)$ & $20(6-26)$ & $1.18(1.06,1.32)$ & 0.003 \\
\hline Ventricular & $2(0-10)$ & $4(0-12)$ & $1.46(1.13,1.89)$ & 0.003 \\
\hline Perimesencephalic SAH location (no. [\%]) & $11(28.2)$ & $1(6.7)$ & $0.16(0.03,1,83)$ & 0.159 \\
\hline Vasospasm (no. [\%] & $17(43.6)$ & $4(26.7)$ & $0.471(0.13,1.74)$ & 0.259 \\
\hline Hydrocephalus (no. [\%]) & $2(5.1)$ & $6(40.0)$ & $12.33(2.13,71.57)$ & 0.005 \\
\hline $\mathrm{DCl}($ no. $[\%])$ & $11(28.2)$ & $4(26.7)$ & $0.45(0.11,1.86)$ & 0.267 \\
\hline \multicolumn{5}{|l|}{ Treatment (no. [\%]) } \\
\hline Clipped aneurysm & $7(17.9)$ & $3(20.0)$ & $1.57(0.25,10.09)$ & 0.634 \\
\hline Coiled aneurysm & $20(51.3)$ & $9(60.0)$ & $1.65(0.37,7.39)$ & 0.513 \\
\hline
\end{tabular}

* The $p$ values were obtained by logistic regression models. Reference categories: female sex, GCS score <8, WFNS grade I, HH grade I, modified Fisher grade 2-3, SAH location: nonperimesencephalic SAH, treatment: nonaneurysmal SAH/no treatment.

$\dagger$ Computational problems emerging from a quasi-complete separation problem due to the small sample size (poor clinical outcome is perfectly determined by $\mathrm{HH}$ grade).

outcome at 3 months (OR 1.29, 95\% CI 1.04-1.60, p = 0.020 ); for each increase of 10 units in the ADC value, an increase of $29 \%$ in the odds of a poor outcome was found.

Additionally, for each unit increase in the cisternal Hijdra grade, there was a $21 \%$ increase in the odds of a poor outcome (OR 1.21, 95\% CI 1.00-1.47, $\mathrm{p}=0.050)$, and for each increase of 1 unit in the GCS score, there was a $53 \%$ decrease in the odds of a poor outcome (OR $0.47,95 \%$ CI $0.30-0.74, \mathrm{p}=0.001)$. The ROC curve had an AUC of 0.95 (95\% CI 0.90-1.00, excellent discriminative ability), and the Hosmer-Lemeshow goodness-of-fit test showed good calibration $(\mathrm{p}=0.960)$.

\section{Discussion}

In this prospective study, we found an association between the FA and ADC values measured in the subacute stage of SAH and clinical outcome. Increased ADC values at the subcortical frontal white matter and reduced FA values at the corpus callosum were independent predictors of a poor outcome at 3 months. In accordance with what has been established in other SAH cohorts, ${ }^{12,31,35,39}$ a higher blood burden on admission CT, lower clinical scores on admission, and patient age were strongly associated with a poor clinical outcome in our patients.

The pathophysiological mechanisms causing cerebral injury in SAH are not fully understood. Immediately after hemorrhage, drastic changes can occur, including an increase in intracranial pressure, the loss of autoregula- tion, cortical spreading depolarization, blood-brain barrier opening, and neuronal apoptosis, leading to early brain injury. ${ }^{32}$ After the initial insult, other complications may ensue-for example, hydrocephalus, vasospasm, and ultimately DCI - and contribute to brain injury and influence the prognosis. Vasospasm, one of the most serious complications of SAH, usually starts after the 4th day, has a peak around 8-10 days, and can persist until around 3 weeks after SAH. ${ }^{8,23}$ Delayed cerebral ischemia is eventually the result of vasospasm, although it is also thought to reflect the early brain injury occurring in the first days after $\mathrm{SAH} .{ }^{31}$

The role of MRI in predicting prognosis in SAH has been addressed, $, 511,37$ with most studies characterizing the diffusion-weighted imaging (DWI) and FLAIR lesion burden and focusing on poor-grade patients, whose prognosis is difficult to determine. Acute ischemic injury after SAH detectable on DWI has been shown to be proportional to neurological impairment on admission ${ }^{5,37}$ and to be associated with long-term outcome. ${ }^{11}$ However, few studies have sought to identify DTI markers of prognosis in SAH, as has been done for other diseases such as traumatic brain injury ${ }^{40}$ and small vessel disease. ${ }^{27}$

Fractional anisotropy and ADC represent different physiological mechanisms. ${ }^{22,26}$ Fractional anisotropy is used as a measure of axonal integrity ${ }^{26,28}$ and can be reduced even without visible lesions on conventional MRI sequences, functioning as a subtle marker of the microstructural integrity of white matter. We found that patients 


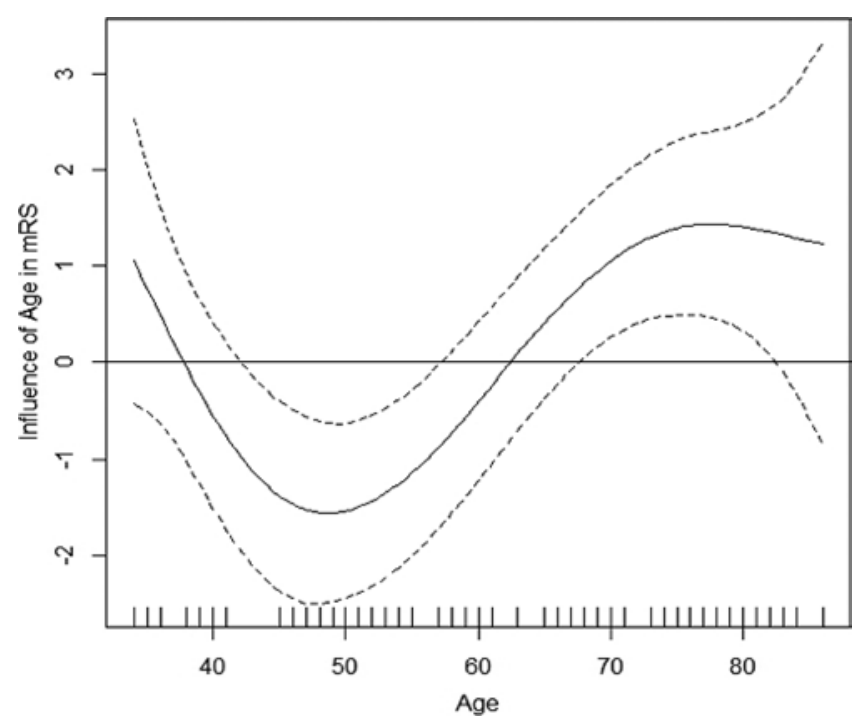

FIG. 3. Influence of age on outcome at 3 months (solid curve) and corresponding 95\% confidence intervals (dashed curves), showing a nonlinear association that led to a dichotomization into 2 categories: age $42-67$ years and ages $<42 />67$ years.

with better outcomes had globally higher FA values (both in individual regions and in whole-brain measurements) and that FA measured at the corpus callosum in the sub- acute phase was an independent predictor of a good clinical outcome. Our results are somewhat in line with those of Sener and colleagues, ${ }^{33}$ who demonstrated, in a cohort of $23 \mathrm{SAH}$ patients, the association between reduced whole-brain FA at 12 days post-SAH and decreased survival at 6 months. However, these results were not adjusted for other variables influencing prognosis. An axonal lesion occurring in SAH-eventually due to early brain injury in the first hours after SAH or secondary to complications after the acute phase such as hydrocephalus, vasospasm, or DCI-may explain these findings: FA will be reduced in patients with more extensive parenchymal injury, and it can be a biomarker of a worse prognosis.

Apparent diffusion coefficient reflects the diffusion of water molecules across cell membranes. Early ischemia leads to cytotoxic edema secondary to an energetic failure at the cell membrane, reducing ADC. ${ }^{7}$ On the contrary, the facilitated diffusion of water molecules in vasogenic edema leads to an increase in ADC. Most studies reporting on early MRI in acute SAH have focused on acute ischemic lesions on DWI, with a corresponding reduced ADC., ${ }^{4,511}$ However, a recent study by Weimer et al. described a global increase in the ADC values in normal-appearing parenchyma as early as the first 72 hours post-SAH, ${ }^{38}$ but these authors did not assess the relation of this finding to prognosis. In a previous study, ${ }^{9}$ we found that early increases in ADC values in normal-appearing white matter, mea-

TABLE 3. Univariable regression analysis results for a poor clinical outcome, considering the DTI parameters obtained on MRI at 8-10 days post-SAH

\begin{tabular}{|c|c|c|c|c|}
\hline Parameter & mRS Score $<3$ & $\mathrm{mRS}$ Score $\geq 3$ & OR Estimate $(95 \% \mathrm{Cl})$ & $\mathrm{p}$ Value \\
\hline No. of patients & 39 & 15 & & \\
\hline \multicolumn{5}{|l|}{$\mathrm{FA}$} \\
\hline FCSO & $0.50(0.07)$ & $0.45(0.07)$ & $0.42(0.17,1.00)$ & 0.049 \\
\hline PCSO & $0.48(0.05)$ & $0.45(0.08)$ & $0.49(0.18,1.29)$ & 0.149 \\
\hline Lentiform nucleus & $0.19(0.05)$ & $0.21(0.07)$ & $2.08(0.68,6.38)$ & 0.200 \\
\hline Thalamus & $0.30(0.05)$ & $0.29(0.05)$ & $0.72(0.20,2.58)$ & 0.613 \\
\hline PLIC & $0.65(0.05)$ & $0.66(0.06)$ & $1.51(0.46,4.98)$ & 0.498 \\
\hline G\&S of corpus callosum & $0.73(0.09)$ & $0.60(0.14)$ & $0.38(0.21,0.69)$ & 0.001 \\
\hline Cerebellum & $0.39(0.06)$ & $0.35(0.07)$ & $0.30(0.10,0.87)$ & 0.026 \\
\hline Mid pons & $0.36(0.11)$ & $0.31(0.11)$ & $0.63(0.34,1.18)$ & 0.147 \\
\hline Whole brain & $0.45(0.05)$ & $0.42(0.04)$ & $0.41(0.01,0.36)$ & 0.004 \\
\hline \multicolumn{5}{|l|}{$\mathrm{ADC}$} \\
\hline FCSO & $706.4(45.5)$ & $738.8(74.0)$ & $1.13(0.99,1.30)$ & 0.071 \\
\hline PCSO & $732.5(49.4)$ & $738.9(62.9)$ & $1.02(0.91,1.15)$ & 0.698 \\
\hline Lentiform nucleus & $725.9(68.2)$ & $779.8(110.4)$ & $1.08(1.00,1.17)$ & 0.053 \\
\hline Thalamus & $756.8(51.7)$ & $821.2(115.4)$ & $1.11(1.02,1.21)$ & 0.019 \\
\hline PLIC & $697.8(47.5)$ & $725.7(71.4)$ & $1.10(0.98,1.24)$ & 0.120 \\
\hline G\&S of corpus callosum & $766.2(93.8)$ & 807.6 (191.7) & $1.03(0.98,1.08)$ & 0.302 \\
\hline Cerebellum & $666.5(42.3)$ & $698.6(66.1)$ & $1.15(1.00,1.32)$ & 0.053 \\
\hline Mid pons & $666.7(57.7)$ & $695.2(47.7)$ & $1.10(0.98,1.23)$ & 0.110 \\
\hline Whole brain & $714.8(34.8)$ & $750.8(55.9)$ & $1.24(1.04,1.47)$ & 0.016 \\
\hline
\end{tabular}

$\mathrm{FCSO}$ = frontal centrum semiovale; $\mathrm{G \& S}=$ genu and splenium; $\mathrm{PCSO}=$ parietal centrum semiovale.

Values expressed as the mean (standard deviation). The $p$ values were obtained using logistic regression models; variables attaining a $p$ value $\leq 0.25$ were considered for multivariable analysis. 

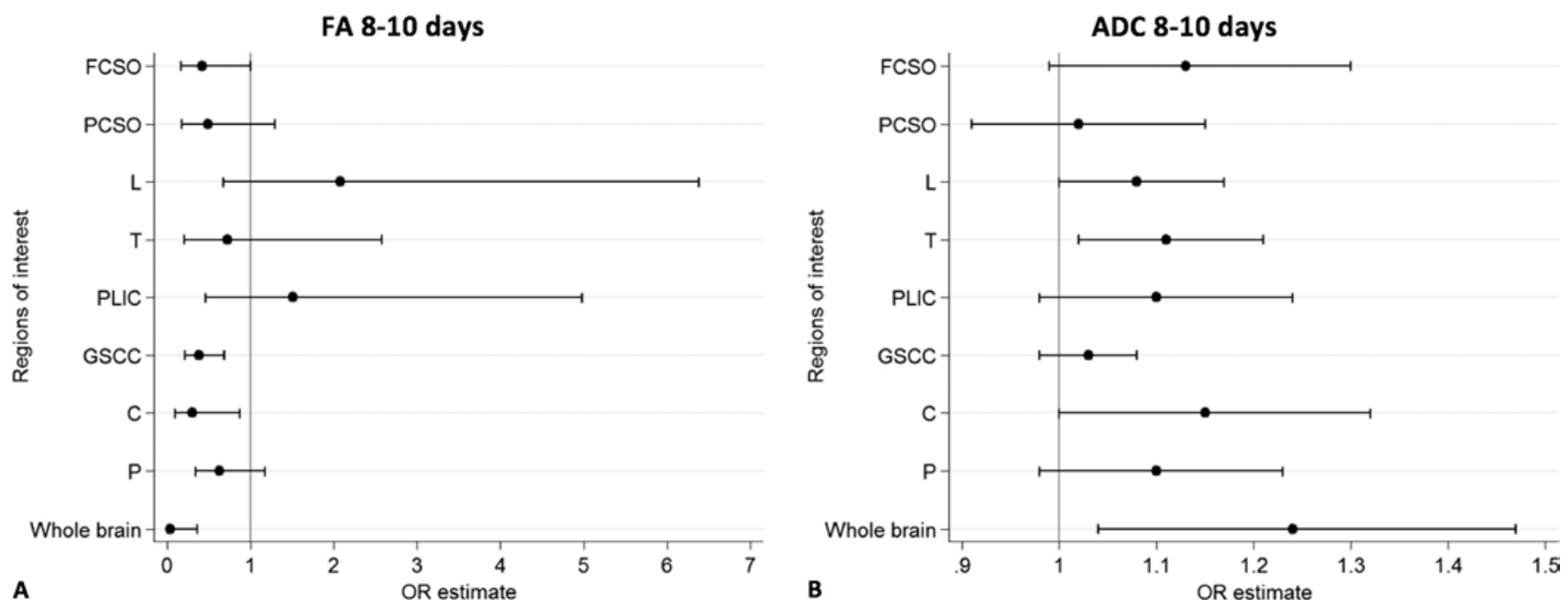

FIG. 4. Odds ratio estimates and corresponding $95 \%$ confidence intervals obtained by logistic regression models for a poor clinical outcome in each ROI. A: Fractional anisotropy at 8-10 days post-SAH. B: Apparent diffusion coefficient at 8-10 days post-SAH. $C=$ cerebellum; $F C S O=$ frontal centrum semiovale; $G S C C=$ genu and splenium of corpus callosum; $L=$ lentiform nucleus; $P=$ pons; $\mathrm{PCSO}=$ parietal centrum semiovale; $\mathrm{T}=$ thalamus.

sured within 72 hours after SAH, were associated with a worse prognosis. According to our present results, these changes also seem to occur in the subacute phase of SAH. Liu et al..$^{25}$ described a mild diffusely increased ADC of the subcortical white matter but did not analyze its impact on prognosis. Our results are in line with their findings and add a prognostic value of the ADC measurements in the subacute phase of $\mathrm{SAH}$, helping to predict the clinical outcome.

The pathophysiological mechanisms explaining this increase in ADC in the subacute phase of SAH possibly start as early as the first 72 hours and are probably multifactorial: altered cerebral autoregulation, effects of treatment (hypervolemia), and disruption of the blood-brain barrier, ${ }^{25}$ the latter peaking at 48 hours post-SAH. ${ }^{14}$ All of these factors together may negatively impact clinical prognosis.

Modifications of FA and ADC reflect different mechanisms, and although we found some significant associations with outcome, the pathophysiological meaning of these associations remains undetermined.

The finding of an association between a younger age and a worse outcome is surprising. Besides the higher frequency of vasospasm in this age group, we hypothesize that the increased intracranial pressure may also be more harmful in younger patients. Older patients, with their higher degree of cerebral atrophy leading to an increase in the extraaxial compartments, seem to better tolerate increases in intracranial pressure, as is known, for example, in malignant edema after ischemic stroke. ${ }^{16}$

We acknowledge some limitations to our study. The small size of the study population probably explains the selective association of certain ROIs since other regions of the parenchyma showed similar associations in the univariable analysis but did not reach statistical significance in the multivariable analysis. Limitations of DTI measurements, mainly affecting FA, are known given that crossing the white matter fibers can modify FA values. ${ }^{28}$ Moreover, the reliability of measurements of DTI parameters may be questioned. However, in a study by Brander et al., ${ }^{3}$ intraobserver agreement was acceptable in most anatomical regions, with the best agreement found at the genu and splenium of the corpus callosum and the lowest agreement at the centrum semiovale.

One of the limitations of this study was that some patients in very poor neurological condition, for example, those with severe vasospasm and a decreased level of consciousness, did not undergo MRI at 8-10 days after SAH. This may have led to a selection bias, meaning that we imaged patients in better clinical condition. On the other hand, this underlines the value of our finding that DTI may provide incremental value in prognostication in these patients.

The lack of a negative control group is a limitation as well. Long-term follow-up MRI would be important to determine if the observed FA and ADC changes persist in time or are limited to the subacute phase of SAH. Moreover, global and anatomical volumetric evaluations of brain structures would be interesting to correlate with DTI measurements since there are described changes in cerebral volume in the chronic stage of SAH. ${ }^{1}$

Finally, to confirm our preliminary results, larger studies and the inclusion of additional patients, such as poorgrade SAH patients, are needed.

\section{Conclusions}

A poor clinical outcome after SAH was independently associated with DTI parameters in specific regions of the brain. Decreased FA and increased ADC may reflect different mechanisms occurring in the subacute phase of $\mathrm{SAH}$, influencing the clinical prognosis of patients. Larger studies are needed to confirm the results of this exploratory study and to establish the utility of DTI as a prognostic tool in SAH. 


\section{Acknowledgments}

Dr. Fragata was supported by a grant from the Sociedade Portuguesa de AVC/Tecnifar.

\section{References}

1. Bendel P, Koivisto T, Aikiä M, Niskanen E, Könönen M, Hänninen T, et al: Atrophic enlargement of CSF volume after subarachnoid hemorrhage: correlation with neuropsychological outcome. AJNR Am J Neuroradiol 31:370-376, 2010

2. Bonita R, Beaglehole R: Modification of Rankin Scale: Recovery of motor function after stroke. Stroke 19:1497-1500, 1988

3. Brander A, Kataja A, Saastamoinen A, Ryymin P, Huhtala $\mathrm{H}$, Ohman J, et al: Diffusion tensor imaging of the brain in a healthy adult population: Normative values and measurement reproducibility at $3 \mathrm{~T}$ and 1.5 T. Acta Radiol 51:800-807, 2010

4. Condette-Auliac S, Bracard S, Anxionnat R, Schmitt E, Lacour JC, Braun M, et al: Vasospasm after subarachnoid hemorrhage: interest in diffusion-weighted MR imaging. Stroke 32:1818-1824, 2001

5. De Marchis GM, Filippi CG, Guo X, Pugin D, Gaffney CD, Dangayach NS, et al: Brain injury visible on early MRI after subarachnoid hemorrhage might predict neurological impairment and functional outcome. Neurocrit Care 22:74-81, 2015

6. Drake CG: Report of World Federation of Neurological Surgeons Committee on a Universal Subarachnoid Hemorrhage Grading Scale. J Neurosurg 68:985-986, 1988 (Letter)

7. Ebisu T, Naruse S, Horikawa Y, Ueda S, Tanaka C, Uto M, et al: Discrimination between different types of white matter edema with diffusion-weighted MR imaging. J Magn Reson Imaging 3:863-868, 1993

8. Fisher CM, Kistler JP, Davis JM: Relation of cerebral vasospasm to subarachnoid hemorrhage visualized by computerized tomographic scanning. Neurosurgery 6:1-9, 1980

9. Fragata I, Alves M, Papoila AL, Nunes AP, Ferreira P, Canto-Moreira N, et al: Early prediction of delayed ischemia and functional outcome in acute subarachnoid hemorrhage. Stroke 48:2091-2097, 2017

10. Fragata I, Canhão P, Alves M, Papoila AL, Canto-Moreira N: Evolution of diffusion tensor imaging parameters after acute subarachnoid haemorrhage: a prospective cohort study. Neuroradiology 59:13-21, 2017

11. Frontera JA, Ahmed W, Zach V, Jovine M, Tanenbaum L, Sehba F, et al: Acute ischaemia after subarachnoid haemorrhage, relationship with early brain injury and impact on outcome: a prospective quantitative MRI study. J Neurol Neurosurg Psychiatry 86:71-78, 2015

12. Frontera JA, Claassen J, Schmidt JM, Wartenberg KE, Temes $\mathrm{R}$, Connolly ESJ Jr, et al: Prediction of symptomatic vasospasm after subarachnoid hemorrhage: the modified fisher scale. Neurosurgery 59:21-27, 2006

13. Frontera JA, Fernandez A, Schmidt JM, Claassen J, Wartenberg KE, Badjatia N, et al: Defining vasospasm after subarachnoid hemorrhage: what is the most clinically relevant definition? Stroke 40:1963-1968, 2009

14. Germanò A, d'Avella D, Imperatore C, Caruso G, Tomasello F: Time-course of blood-brain barrier permeability changes after experimental subarachnoid haemorrhage. Acta Neurochir (Wien) 142:575-581, 2000

15. Hackett ML, Anderson CS: Health outcomes 1 year after subarachnoid hemorrhage: an international population-based study. Neurology 55:658-662, 2000

16. Heiss WD: Malignant MCA infarction: pathophysiology and imaging for early diagnosis and management decisions. Cerebrovasc Dis 41:1-7, 2016
17. Helbok R, Kurtz P, Vibbert M, Schmidt MJ, Fernandez L, Lantigua $\mathrm{H}$, et al: Early neurological deterioration after subarachnoid haemorrhage: risk factors and impact on outcome. J Neurol Neurosurg Psychiatry 84:266-270, 2013

18. Hijdra A, Brouwers PJ, Vermeulen M, van Gijn J: Grading the amount of blood on computed tomograms after subarachnoid hemorrhage. Stroke 21:1156-1161, 1990

19. Hop JW, Rinkel GJE, Algra A, van Gijn J: Case-fatality rates and functional outcome after subarachnoid hemorrhage: a systematic review. Stroke 28:660-664, 1997

20. Hunt WE, Hess RM: Surgical risk as related to time of intervention in the repair of intracranial aneurysms. J Neurosurg 28:14-20, 1968

21. Janssen PM, Visser NA, Dorhout Mees SM, Klijn CJM, Algra A, Rinkel GJE: Comparison of telephone and face-toface assessment of the modified Rankin Scale. Cerebrovasc Dis 29:137-139, 2010

22. Jellison BJ, Field AS, Medow J, Lazar M, Salamat MS, Alexander AL: Diffusion tensor imaging of cerebral white matter: a pictorial review of physics, fiber tract anatomy, and tumor imaging patterns. AJNR Am J Neuroradiol 25:356-369, 2004

23. Kistler JP, Crowell RM, Davis KR, Heros R, Ojemann RG, Zervas T, et al: The relation of cerebral vasospasm to the extent and location of subarachnoid blood visualized by CT scan: a prospective study. Neurology 33:424-436, 1983

24. Lindegaard KF, Nornes H, Bakke SJ, Sorteberg W, Nakstad P: Cerebral vasospasm diagnosis by means of angiography and blood velocity measurements. Acta Neurochir (Wien) 100:12-24, 1989

25. Liu Y, Soppi V, Mustonen T, Könönen M, Koivisto T, Koskela A, et al: Subarachnoid hemorrhage in the subacute stage: elevated apparent diffusion coefficient in normal-appearing brain tissue after treatment. Radiology 242:518-525, 2007

26. Mori S, Zhang J: Principles of diffusion tensor imaging and its applications to basic neuroscience research. Neuron 51:527-539, 2006

27. Nitkunan A, Barrick TR, Charlton RA, Clark CA, Markus HS: Multimodal MRI in cerebral small vessel disease: its relationship with cognition and sensitivity to change over time. Stroke 39:1999-2005, 2008

28. Nucifora PGP, Verma R, Lee SK, Melhem ER: Diffusiontensor MR imaging and tractography: exploring brain microstructure and connectivity. Radiology 245:367-384, 2007

29. Pegoli M, Mandrekar J, Rabinstein AA, Lanzino G: Predictors of excellent functional outcome in aneurysmal subarachnoid hemorrhage. J Neurosurg 122:414-418, 2015

30. Rinkel GJE, Algra A: Long-term outcomes of patients with aneurysmal subarachnoid haemorrhage. Lancet Neurol 10:349-356, 2011

31. Rosengart AJ, Schultheiss KE, Tolentino J, Macdonald RL: Prognostic factors for outcome in patients with aneurysmal subarachnoid hemorrhage. Stroke 38:2315-2321, 2007

32. Sehba FA, Pluta RM, Zhang JH: Metamorphosis of subarachnoid hemorrhage research: from delayed vasospasm to early brain injury. Mol Neurobiol 43:27-40, 2011

33. Sener S, Van Hecke W, Feyen BFE, Van der Steen G, Pullens P, Van de Hauwe L, et al: Diffusion tensor imaging: a possible biomarker in severe traumatic brain injury and aneurysmal subarachnoid hemorrhage? Neurosurgery 79:786-793, 2016

34. Teasdale G, Jennett B: Assessment of coma and impaired consciousness. A practical scale. Lancet 2:81-84, 1974

35. van Gijn J, Hijdra A, Wijdicks EF, Vermeulen M, van Crevel $\mathrm{H}$ : Acute hydrocephalus after aneurysmal subarachnoid hemorrhage. J Neurosurg 63:355-362, 1985

36. Vergouwen MDI, Vermeulen M, van Gijn J, Rinkel GJ, Wijdicks EF, Muizelaar JP, et al: Definition of delayed cerebral 
ischemia after aneurysmal subarachnoid hemorrhage as an outcome event in clinical trials and observational studies: proposal of a multidisciplinary research group. Stroke 41:2391-2395, 2010

37. Wartenberg KE, Sheth SJ, Michael Schmidt J, Frontera JA, Rincon F, Ostapkovich N, et al: Acute ischemic injury on diffusion-weighted magnetic resonance imaging after poor grade subarachnoid hemorrhage. Neurocrit Care 14:407415,2011

38. Weimer JM, Jones SE, Frontera JA: Acute cytotoxic and vasogenic edema after subarachnoid hemorrhage: a quantitative MRI study. AJNR Am J Neuroradiol 38:928-934, 2017

39. Witsch J, Frey HP, Patel S, Park S, Lahiri S, Schmidt JM, et al: Prognostication of long-term outcomes after subarachnoid hemorrhage: the FRESH score. Ann Neurol 80:46-58, 2016

40. Yuh EL, Cooper SR, Mukherjee P, Yue JK, Lingsma HF, Gordon WA, et al: Diffusion tensor imaging for outcome prediction in mild traumatic brain injury: a TRACK-TBI study. $\mathbf{J}$ Neurotrauma 31:1457-1477, 2014

\section{Disclosures}

The authors report no conflict of interest concerning the materials or methods used in this study or the findings specified in this paper.

\section{Author Contributions}

Conception and design: Fragata, Canhão. Acquisition of data: Fragata, Ferreira, Nunes. Analysis and interpretation of data: Fragata, Alves, Papoila, Canhão. Drafting the article: Fragata, Alves, Papoila. Critically revising the article: Alves, Papoila, CantoMoreira, Canhão. Reviewed submitted version of manuscript: all authors. Approved the final version of the manuscript on behalf of all authors: Fragata. Statistical analysis: Alves, Papoila. Study supervision: Fragata, Canhão.

\section{Correspondence}

Isabel Fragata: Centro Hospitalar Lisboa Central, Lisbon, Portugal. isabelfragata@gmail.com. 